\title{
Picosecond infrared probing of the vibrational spectra of transients formed upon UV excitation of stacked G-tetrad structures $\uparrow$
}

\author{
David A. McGovern, ${ }^{a}$ Susan Quinn, ${ }^{* a}$ Gerard W. Doorley, ${ }^{a}$ Aine M. Whelan, ${ }^{a}$ Kate L. Ronayne, ${ }^{b}$ \\ Michael Towrie, ${ }^{b}$ Anthony W. Parker*b and John M. Kelly*a
}

Received (in Cambridge, UK) 23rd July 2007, Accepted 30th August 2007

First published as an Advance Article on the web 13th September 2007

DOI: $10.1039 / \mathrm{b} 711172 \mathrm{j}$

The photophysical properties of stacked G-tetrads in diverse systems, including concentrated solutions of 5'-guanosine monophosphate (5'-GMP), polyguanylic acid (poly(G)) and the G-rich oligodeoxynucleotide sequence characteristic of human telomeric DNA, are probed by ps-TRIR and compared to those of the monomeric 5'-GMP.

Electronic excited states of individual nucleic acid bases and nucleotides are typically characterised by ultrafast relaxation processes $(<1 \mathrm{ps}) .^{1-3}$ However, in higher order structures, such as polymeric strands and aggregated species, longer-lived transients are observed, ${ }^{2 a}$ and these present a means to promote chemical modifications leading to genetic mutation. The proposed mechanisms for these extended lifetimes include base-stacking interactions, excimer/exciplex formation and hydrogen bonding. ${ }^{4}$ To fully understand the nature of the molecular specific interactions, it is essential to employ techniques that can interrogate the structural modifications underpinning these processes, and here we apply ultrafast time-resolved infrared spectroscopy (TRIR) for this purpose. ps-TRIR is now being demonstrated as a powerful technique for probing the photophysical properties of DNA, ${ }^{5-8}$ and its strength stems from its ability to yield kinetic and structural information that goes beyond that obtainable from fluorescence or transient UV/visible absorption techniques.

In this communication, we describe how ps-TRIR can be employed to investigate conformationally dependent photophysical properties of base-base interactions. Guanine is known to aggregate to form stacked species at high concentrations. ${ }^{9}$ It is also interesting as this base absorbs more strongly at longer wavelengths and is the most sensitive of the nucleobases to singlet oxygen and photodynamic oxidation. In this paper, we initially consider 5'-GMP at concentrations where it is (a) monomeric and (b) stacked. These results are then compared to the polymer $\operatorname{poly}(\mathrm{G})$ (which is known to spontaneously form structures composed of stacked guanine tetrads) and, finally, to the biologically relevant G-rich telomeric sequence dAGGG(TTAGGG) $)_{3}$ which is

${ }^{a}$ School of Chemistry and Centre for Chemical Synthesis and Chemical Biology,Trinity College,Dublin 2,Ireland.E-mail: jmkelly@tcd.ie; quinnsu@tcd.ie; Fax: +353 16712826; Tel: +35318961947

${ }^{b}$ Central Laser Facility, Science \& Technology Facilities Council

Rutherford Appleton Laboratory, Harwell Science and Innovation

Campus, Didcot, Oxfordshire, UK OX11 0QX.

E-mail:A.W.Parker@rl.ac.uk

$\dagger$ Electronic supplementary information (ESI) available: Method for ps-

TRIR measurements, sample preparation and simulation studies. See

DOI: $10.1039 / \mathrm{b} 711172 \mathrm{j}$ vital for protection against genome degradation and plays an important role in aging and cancer. ${ }^{10}$

Fig. 1a shows the ps-TRIR spectra of a neutral solution of 5'-GMP (10 mM, pH 7.0) at various delays after excitation with $267 \mathrm{~nm}$ radiation. The spectrum contains regions of transient absorption and regions where depletion ('bleaching') of the ground state bands is dominant. The 5'-GMP bleaches occur at 1581 and $1669 \mathrm{~cm}^{-1}$ and are attributed predominantly to ground state ringbased and carbonyl vibrations respectively. ${ }^{11}$ The shifting of the transient absorption bands to higher wavenumbers in the first $\sim 5$ ps is consistent with cooling of the vibrationally hot ground state following electronic excitation. ${ }^{5}$ This band-shifting coupled with spectral overlap of the ground state and transient bands means that kinetics obtained from intensity changes at a single pixel position produces a distribution of decay/recovery times across the band profiles (data given in ESI $\dagger$ ). In order to resolve this complexity and obtain reliable kinetics, we have modelled the

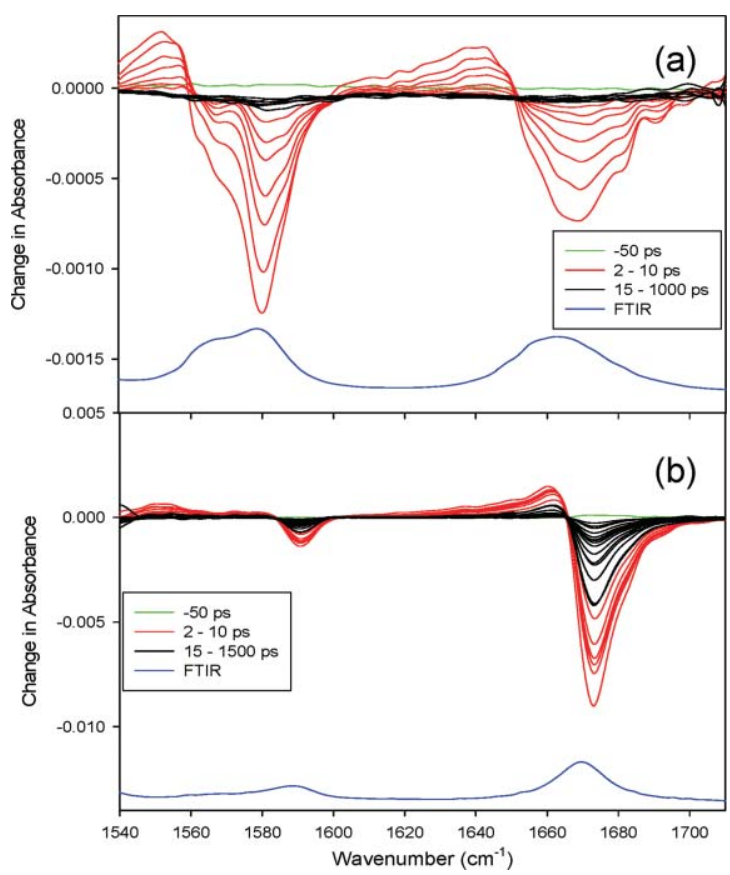

Fig. 1 FTIR and ps-TRIR spectra recorded at delays of -50 (green), 2, $3,4,6.5,10,15,20,35,50,100,500,1000$ ps (both (a) and (b)), 1250, $1500 \mathrm{ps}$ ((b) only) after excitation at $267 \mathrm{~nm}$ of (a) $10 \mathrm{mM} \mathrm{Na}_{2}$ GMP in $50 \mathrm{mM}$ sodium phosphate buffer in $\mathrm{D}_{2} \mathrm{O}, \mathrm{pH} 7.0$; (b) $540 \mathrm{mM} \mathrm{K} \mathrm{K}_{2} \mathrm{GMP}$ and $540 \mathrm{mM}$ potassium chloride in $\mathrm{D}_{2} \mathrm{O}$. Red traces are at delays less than or equal to 10 ps. FTIR spectra are in blue and appear along the $x$-axis. 
experimental spectral profile using overlapping transient and depletion Gaussian bands for both the carbonyl and ring-based vibrations. The experimental data reveal recovery times of $4.7 \pm$ $0.5 \mathrm{ps}$ and $3.1 \pm 0.3 \mathrm{ps}$ for the ring and carbonyl bleaches respectively. This modelling, combined with the stabilising of the distribution towards the higher wavenumber side of both bleaches where the influence of the recovering associated transient is minimal, supports the experimental findings. The fact that the ring mode recovers on a longer timescale than the carbonyl modes is indicative of mode-specific relaxation within the guanine system and is supported by recent work of Zanni et al., which shows that the $\mathrm{C}=\mathrm{N}$ ring stretching and $\mathrm{C}=\mathrm{O}$ carbonyl stretching modes are not strongly coupled. ${ }^{12}$ This result may also be a consequence of increased interaction of the carbonyl mode with the solvent molecules.

In solution, 5'-GMP self-associates in the presence of alkali metals to form stacked G-tetrad structures, ${ }^{13}$ which X-ray diffraction reveals to be fibrous in nature. ${ }^{14}$ Both the carbonyl and ringstretching vibrations are very sensitive to the formation of such hydrogen-bonded and base-stacked guanine-containing species. The FTIR reveals significant changes in these bands. The ring vibrational stretch is found to undergo strong hypochromism and to shift from 1581 to $1590 \mathrm{~cm}^{-1}$, while the carbonyl band narrows and moves to higher wavenumbers from 1669 to $1673 \mathrm{~cm}^{-1} .15$ This shift in wavenumber has been observed for similar guanine systems and may be attributed to the formation and stacking of guanine tetrads. ${ }^{16}$ The sharpening of the ground state bands probably indicates a narrowing in the range of conformers of the guanine moiety within the higher ordered structure. To observe the effect of stacking on the transient spectra of these species, the ps-TRIR was measured under conditions (540 $\mathrm{mM} \mathrm{K}_{2} \mathrm{GMP}$, $540 \mathrm{mM} \mathrm{KCl}$ ) known to stabilize higher order structures (Fig. 1b). ${ }^{17}$ It may be noted that the behaviour observed is very different to that of monomeric 5'-GMP. The ring-based vibrations at $1590 \mathrm{~cm}^{-1}$ are noticeably suppressed and the carbonyl vibration at $1673 \mathrm{~cm}^{-1}$ now dominates and is substantially sharper, mirroring the ground state FTIR of the system. The relative narrowness of the feature in the difference spectrum is also a consequence of the transient absorption, which is sharper and has a maximum less displaced from the ground state than in the dilute solutions of $5^{\prime}$-GMP, and we interpret this as meaning that there is little nuclear rearrangement occurring within the excited state and that $\mathrm{H}$-bonding interactions remain largely unperturbed.

The kinetic behaviour of $540 \mathrm{mM} 5^{\prime}-\mathrm{K}_{2} \mathrm{GMP}$ reveals the presence of a longer-lived state (Fig. 2). The recovery of the carbonyl bleach at $1673 \mathrm{~cm}^{-1}$ shows a biexponential process with a short component of $4 \pm 1$ ps $(60 \%)$ and a longer component of $49 \pm 9$ ps $(40 \%)$. Similar lifetimes were obtained for the ring bleach and the corresponding transient species. We attribute the short lifetime to vibrational relaxation of the ground state as was observed in dilute solution. The longer lived (49 ps) species is tentatively attributed to an electronic excited state species (probably an excimer). ${ }^{2 a}$

Extending this system further, we next examined the ps-TRIR of polyguanylic acid $(\operatorname{poly}(\mathrm{G}))$ at a low total nucleotide concentration (11.4 mM nucleotide, $\mathrm{pH} 7.0$ ), similar to that used for the non-aggregated 5'-GMP system. Strikingly, the transient difference spectra (Fig. 3a) are very similar to those of the stacked G-tetrads at the higher concentration of 5'-GMP (Fig. 1b). Again

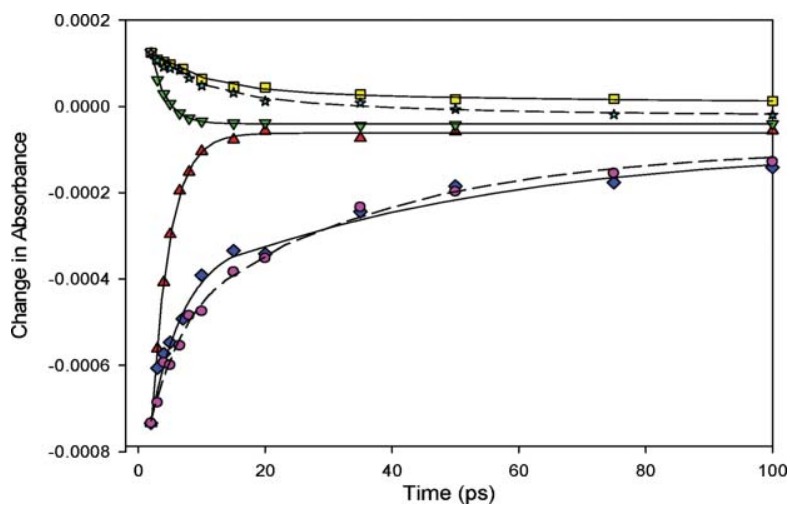

Fig. 2 Kinetics recorded at the carbonyl bleach and associated transient positions for: $10 \mathrm{mM} \mathrm{Na}_{2} \mathrm{GMP}: 1676 \mathrm{~cm}^{-1}(\mathbf{\Delta}), 1640 \mathrm{~cm}^{-1}(\boldsymbol{\nabla}) ; 540 \mathrm{mM}$ $\mathrm{K}_{2} \mathrm{GMP}$ in $540 \mathrm{mM} \mathrm{KCl}: 1673 \mathrm{~cm}^{-1}(\diamond), 1660 \mathrm{~cm}^{-1}(\boldsymbol{\square})$; and $11.4 \mathrm{mM}$ poly(G): $1687 \mathrm{~cm}^{-1}(\bullet), 1658 \mathrm{~cm}^{-1}(\star)$.

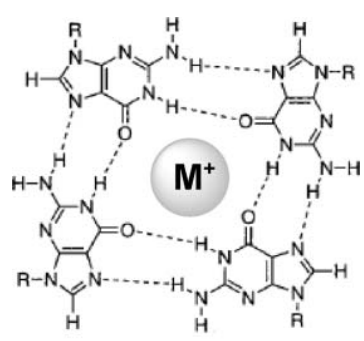

Guanine Tetrad

there is suppression of ring-based vibrations and a sharpening of the carbonyl vibration which shifts from 1669 to $1690 \mathrm{~cm}^{-1}$, reflecting the ground state FTIR of the system and attributable to the formation and stacking of guanine tetrads. ${ }^{16}$ The kinetic behaviour of the poly $(\mathrm{G})$ also differs markedly from that of 5'-GMP in dilute solution but is similar to that of the stacked system formed in the presence of high cation concentration. (Fig. 2) The recovery of the carbonyl bleach at $1687 \mathrm{~cm}^{-1}$ reveals a biexponential process with a short component of $5 \pm 1$ ps $(47 \%)$ and a longer component of $37 \pm 6$ ps $(53 \%)$. As with dilute 5'-GMP, the short lifetime may be attributed to vibrational relaxation of the ground state, while the longer-lived species can be assigned to an excited state (probably an excimer). ${ }^{2 a}$ Similar kinetics were obtained for the transient species as for the bleach recovery.

Having considered the effect of stacking interactions on the lifetimes of 5'-GMP in monomeric and polymeric assembled systems, we next wished to probe such interactions in a biologically relevant G-rich system. For this study, an oligodeoxynucleotide containing the tandem repeat human telomer sequence dAGGG(TTAGGG) ${ }_{3}$ sequence was chosen. This is known to form a quadruplex structure in the presence of cations. ${ }^{18} \mathrm{~A}$ sample of $10 \mathrm{mM} \mathrm{dAGGG(TTAGGG)})_{3}$ in the presence of $100 \mathrm{mM} \mathrm{KCl}$ was annealed and cooled gradually to room temperature to ensure formation of the quadruplex structure, which was confirmed by the presence in the FTIR of a band at $1537 \mathrm{~cm}^{-1} \cdot{ }^{19}$ Consideration of the ps-TRIR spectra (Fig. 3b) shows regions associated with the guanine bleaches, with the carbonyl band dominating at $1672 \mathrm{~cm}^{-1}$ and the ring-centred guanine stretch present at $1591 \mathrm{~cm}^{-1}$, in a similar fashion to that seen for neutral poly $(\mathrm{G})$. Detailed analysis of the kinetics is complicated by additional 


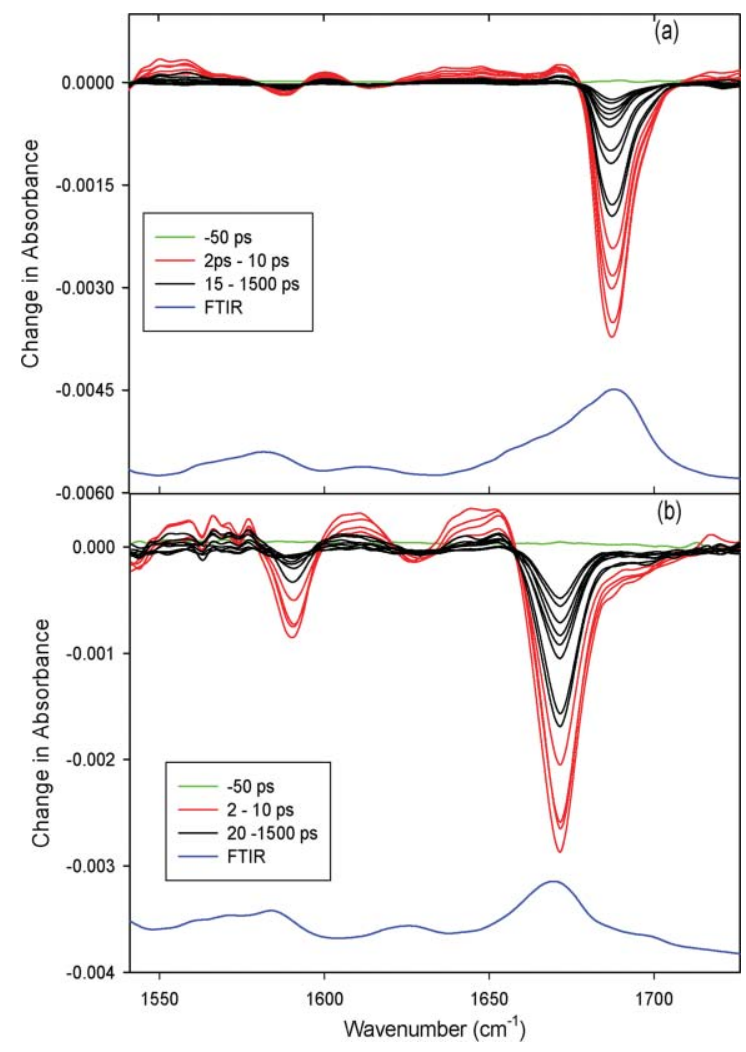

Fig. 3 FTIR and ps-TRIR spectra recorded at (a) delays of -50 (green), $2,3,4,6.5,10,15,20,35,50,100,150,300,500,1000,1500$ ps after excitation at $267 \mathrm{~nm}$ of $11.4 \mathrm{mM}$ poly $(\mathrm{G})$ in $50 \mathrm{mM}$ potassium phosphate buffer in $\mathrm{D}_{2} \mathrm{O}$ at $\mathrm{pH} 7$ and (b) delays of -50 (green), 2, 4, 7, 10, 20, 50, $100,150,200,500,1000,1500 \mathrm{ps}$ after excitation at $267 \mathrm{~nm}$ for $10 \mathrm{mM}$ dAGGG(TTAGGG) $)_{3}$ in $100 \mathrm{mM}$ potassium chloride and $50 \mathrm{mM}$ potassium phosphate buffer in $\mathrm{D}_{2} \mathrm{O}$. Red traces are at delays less than or equal to 10 ps. FTIR spectra are in blue and appear along the $x$-axis.

contributions from the adenine and thymine bases present in the telomeric sequence. The data has been fitted to a biexponential process similar to that observed for the stacked guanine system, although it is notable that the extent of the carbonyl bleach recovery is significantly less reversible on the ps-timescale than in the other systems studied. This long-lived species may be caused by the reactions of thymine moieties in the telomere sequence. ${ }^{8}$ The sharper spectral features in comparison to the free base (Fig. 1a) again suggest the higher-ordered structure of the ground state and transients.

In conclusion, it has been shown that time-resolved infrared spectroscopy can be used to track the evolution of long-lived states from simple guanine systems to aggregated and higher-order ones.
It may be noted that the guanine tetrad structures lead to difference spectra with characteristically sharper features, presumably due to the greater order in these aggregated systems. This order is present in the transient as well as in the ground state species and demonstrates that electronic excitation does not lead to a large scale reordering of the structure. It is hoped that use of this technique to probe other regions of the infrared spectrum will provide more detailed information on structural changes in the nucleotides upon electronic excitation.

We thank EU FP 6 co-ordinated through LaserLabs Europe (CLF001281) for granting access to the Central Laser Facility, STFC Rutherford Appleton Laboratory. We thank the Science Foundation Ireland for financial support and Professor Mike George for useful discussions on all aspects of ps-TRIR.

\section{Notes and references}

1 M. Daniels and W. Hauswirth, Science, 1971, 171, 675.

2 (a) C. E. Crespo-Hernández, B. Cohen, P. M. Hare and B. Kohler, Chem. Rev., 2004, 104, 1977; (b) J. M. L. Pecourt, J. Peon and B. Kohler, J. Am. Chem. Soc., 2001, 123, 10370.

3 (a) A. Sharonov, T. Gustavsson, V. Carré, E. Renault and D. Markovitsi, Chem. Phys. Lett., 2003, 380, 173; (b) D. Onidas, D. Markovitsi, S. Marguet, A. Sharonov and T. Gustavsson, J. Phys. Chem. B, 2002, 106, 11367.

4 C. E. Crespo-Hernández, B. Cohen and B. Kohler, Nature, 2005, 436, 1141.

5 M. K. Kuimova, J. Dyer, M. W. George, D. C. Grills, J. M. Kelly, P. Matousek, A. W. Parker, X. Z. Sun, M. Towrie and A. M. Whelan, Chem. Commun., 2005, 1182.

6 M. K. Kuimova, A. J. Cowan, P. Matousek, A. W. Parker, X. Z. Sun, M. Towrie and M. W. George, Proc. Natl. Acad. Sci. U. S. A., 2006, 103, 2150.

7 S. Quinn, G. W. Doorley, G. W. Watson, A. J. Cowan, M. W. George, A. W. Parker, K. L. Ronayne, M. Towrie and J. M. Kelly, Chem. Commun., 2007, 2130.

8 W. J. Schreier, T. E. Schrader, F. O. Koller, P. Gilch, C. E. CrespoHernández, V. N. Swaminathan, T. Carell, W. Zinth and B. Kohler, Science, 2007, 315, 625.

9 J. T. Davis, Angew. Chem., Int. Ed., 2004, 43, 668.

10 J. W. Shay and W. E. Wright, Cancer Cell, 2002, 2, 257.

11 H. T. Miles, F. B. Howard and J. Frazier, Science, 1963, 142, 1458.

12 A. T. Krummel, P. Mukherjee and M. T. Zanni, J. Phys. Chem. B, 2003, 107, 9165 .

13 T. J. Pinnavaia, C. L. Marshall, C. M. Mettler, C. L. Fisk, H. T. Miles and E. D. Becker, J. Am. Chem. Soc., 1978, 100, 3625.

14 E. A. Lesnik, I. M. Kochkina, A. S. Tikhonenko and Ya. M. Varshavskii, Mol. Biol. (Moscow), 1980, 14, 820.

15 R. Ghana, C. Walss and J. A. Walmsley, J. Biomol. Struct. Dyn., 1996, 14, 101.

16 M. Romero Guzmán, J. Liquier, S. K. Brahmachari and E. Taillandier, Spectrochim. Acta, Part A, 2006, 64, 495.

17 J. A. Walmsley and J. F. Burnett, Biochemistry, 1999, 38, 14063.

18 G. N. Parkinson, M. P. H. Lee and S. Neidle, Nature, 2002, 417, 876.

19 J. A. Mondrágon-Sánchez, J. Liquier, R. H. Shafer and E. Taillandier, J. Biomol. Struct. Dyn., 2004, 22, 365. 